\title{
Acoustic echo cancellation using adaptive filtering algorithms for Quranic accents (Qiraat) identification
}

\begin{abstract}
Echoed parts of Quranic accent (Qiraat) signals are exposed to reverberation of signals especially if they are listened to in a conference room or the Quranic recordings found in different media such as the web. Quranic verse rules identification/Tajweed are prone to additive noise and may reduce classification results. This research work aims to present our work towards Quranic accents (Qiraat) identification, which emphasizes on acoustic echo cancellation (AEC) of all echoed Quranic signals during the preprocessing phase of the system development. In order to conduct the AEC, three adaptive algorithms known as affine projection (AP), least mean square (LMS), and recursive least squares (RLS) are used during the preprocessing phase. Once clean Quranic signals are produced, they undergo feature extraction and pattern classification phases. The Mel Frequency Cepstral Coefficients is the most widely used technique for feature extraction and is adopted in this research work, whereas probabilities principal component analysis (PPCA), K-nearest neighbor (KNN) and gaussian mixture model (GMM) are used for pattern classification. In order to verify our methodology, audio files have been collected for Surat Ad-Duhaa for five different Quranic accents (Qiraat), namely: (1) Ad-Duri, (2) Al-Kisaie, (3) Hafs an A'asem, (4) IbnWardan, and (5) Warsh. Based on our experimental results, the AP algorithm achieved $93.9 \%$ accuracy rate against all pattern classification techniques including PPCA, KNN, and GMM. For LMS and RLS, the achieved accuracy rates are different for PPCA, KNN, and GMM, whereby LMS with PPCA and GMM achieved the same accuracy rate of $96.9 \%$; however, LMS with KNN achieved $84.8 \%$. In addition, RLS with PPCA and GMM achieved the same accuracy rate of $90.9 \%$; however, RLS with KNN achieved $78.8 \%$. Therefore, the AP adaptive algorithm is able to reduce the echo of Quranic accents (Qiraat) signals in a consistent manner against all pattern classification techniques.
\end{abstract}

Keywords: Adaptive filtering; Acoustic echo cancellation; Recursive least squares; Least mean squares; Affine projection; K-nearest neighbour; Probabilities principal component analysis; Gaussian mixture model; Quranic accents (Qiraat) 